\title{
Multi-client Predicate-only Encryption for Conjunctive Equality Tests
}

\author{
Tim van de Kamp ${ }^{1}$ \\ Maarten H. Everts ${ }^{1,2}$ \\ Andreas Peter ${ }^{1}$ \\ Willem Jonker ${ }^{1}$ \\ ${ }^{1}$ University of Twente ${ }^{2}$ TNO \\ \{t.r.vandekamp, a.peter, maarten.everts, w.jonker\}@utwente.nl
}

\begin{abstract}
We propose the first multi-client predicate-only encryption scheme capable of efficiently testing the equality of two encrypted vectors. Our construction can be used for the privacypreserving monitoring of relations among multiple clients. Since both the clients' data and the predicates are encrypted, our system is suitable for situations in which this information is considered sensitive. We prove our construction plaintext and predicate private in the generic bilinear group model using random oracles, and secure under chosen-plaintext attack with unbounded corruptions under the symmetric external Diffie-Hellman assumption. Additionally, we provide a proof-of-concept implementation that is capable of evaluating one thousand predicates defined over the inputs of ten clients in less than a minute on commodity hardware.
\end{abstract}

Keywords: multi-client functional encryption · predicate-only encryption $\cdot$ privacy-preserving multi-client monitoring

\section{Introduction}

Predicate encryption (PE) KSW08 is a special type of encryption that supports the evaluation of functions on encrypted data. On a conceptual level, in predicate encryption a ciphertext of a message $m$ is associated with a descriptive value $x$ and a decryption key $\mathrm{SK}_{f}$ with a predicate $f$. The decryption of

This is the full version of the paper appearing in the procedings of CANS 2017. The final publication is currently in press and will be published by Springer. 
a ciphertext using a key $\mathrm{SK}_{f}$ only succeeds if the predicate $f(x)$ evaluates to TRUE. Special-purpose variants of this notion include identity-based encryption (IBE) [BF01], attribute-based encryption (ABE) [SW05], and hidden vector encryption (HVE) BW07. Another variant of PE is predicate-only encryption KSW08; SSW09]. In predicate-only encryption, ciphertexts do not contain a message $m$, but merely consist of an encryption of the descriptive value $x$. In this case, the decryption algorithm returns the outcome of the predicate $f$ evaluated on the predicate subject $x$, that is, $f(x)$.

The concept of PE can be generalized to functional encryption (FE) ONe11; BSW11, in which the decryption of a ciphertext using a key $\mathrm{SK}_{f}$ for a (not necessarily predicate) function $f$ does not return the original plaintext $m$, but the value $f(m)$ instead. More recently, Goldwasser et al. $\left[\mathrm{GGG}^{+} 14\right.$ formally defined multi-client functional encryption (MC-FE). MC-FE is a type of secret key encryption in which $n$ distinct clients can individually encrypt a message $m_{i}$ using their secret encryption key usk $_{i}$. Using a decryption key for an $n$-ary function $f$, the decryption algorithm takes as input the $n$ ciphertexts of the clients and returns $f\left(m_{1}, \ldots, m_{n}\right)$. Although $\mathrm{FE}$ for generalized functionalities $\mathrm{GGH}^{+} 13$; $\mathrm{GGG}^{+} 14$ is an active field of research and of great theoretical interest, FE constructions for a restricted family of functions (such as predicates) are often far more efficient than FE schemes for arbitrary polynomially sized circuits. For example, most works in the area of MC-FE for generalized functionalities rely on inefficient primitives such as indistinguishability obfuscation or multilinear maps.

In this work, we propose the first multi-client predicate-only encryption scheme. Our construction can evaluate an $n$-ary predicate $f$ on the descriptive values $x_{i}$ coming from $n$ distinct clients. The type of predicates that we can evaluate using our construction is restricted to conjunctive equality tests. To put it simply, our multi-client predicate-only encryption (MC-PoE) scheme is capable of testing the equality of two encrypted vectors. One of these vectors is determined by the decryption key, while the other vector is composed of ciphertexts from several distinct clients. We also provide an extension to our construction in which the decryption keys may contain wildcard components. A wildcard component in the decryption key indicates that it does not matter what the client corresponding to that vector component encrypts: any value matches the wildcard. An attentive reader familiar with the concept of HVE BW07] will recognize the functional similarity between the two concepts. However, a crucial difference in our construction is that the ciphertext vector is composed of the ciphertexts from multiple clients, instead of being generated by a single party. A further comparison of related work is discussed in Section 1.2 .

Our multi-client predicate-only encryption construction uses pairing-based cryptography and satisfies two distinct security notions. The first notion cov- 
ers both the attribute-hiding [KSW08] (also termed plaintext-privacy [SSW09]) and predicate-privacy [SSW09] properties of predicate encryption. Informally, these properties guarantee that an adversary can neither learn the value $x$ of a ciphertext, nor learn the predicate from a given decryption key. Since we construct a multi-client scheme, we choose to adapt the established MC-FE security requirement $\mathrm{GGG}^{+} 14$ for our full security notion of multi-client predicate-only encryption. This full security notion protects against an attacker that has oracle access to both the key generation algorithm and the encryption algorithm. In the associated security game, the adversary is additionally allowed to statically corrupt clients. We prove our construction secure in the generic bilinear group model using random oracles. We also propose the (intuitively weaker) chosenplaintext security notion, in which an attacker has only oracle access to the encryption algorithm, but can instead corrupt an unbounded number of clients. We prove our construction secure under this second notion in the standard model using the symmetric external Diffie-Hellman (SXDH) assumption.

Our construction is designed to be simple and fast. We have implemented and analyzed our construction to evaluate whether it is efficient enough to run in practice. In our proof-of-concept implementation, clients can encrypt their values in about $2.6 \mathrm{~ms}$, while decryption keys, depending on the number of vector components, can be created in less than a second. The Test algorithm, used to evaluate the predicate on the multiple inputs, scales linearly in the number of inputs and requires only 0.10 seconds for the comparison of vectors of length 20 .

\subsection{Motivating Use Cases}

Privacy-preserving monitoring over encrypted data is one of the main applications for multi-client predicate-only encryption. For example, consider the monitoring of a system comprised of various independent subsystems. We want to raise an alarm when a dangerous combination of events at the various subsystems occurs. By centrally collecting status messages of the individual systems, we can check for such situations. Such a central collection of status messages additionally avoids the need for costly interactions between the various systems. However, if these status messages are considered sensitive, the monitoring cannot be done on the cleartext messages. Multi-client predicate-only encryption overcomes this problem by allowing a monitor to evaluate an $n$-ary predicate over multiple ciphertexts and raise an alarm when the predicate returns TRUE.

A careful reader might realize that encryption of the status messages is not a sufficient requirement. If the monitor can check arbitrary predicates, it can as well recover the individual plaintext status messages ${ }^{1}$ making its encryption

\footnotetext{
${ }^{1}$ For example, the monitor could create a decryption key for a predicate evaluation of a
} 
useless. Therefore, we have to require that another party issues the decryption keys to the monitor. Since we can consider the monitor to be a third party, it is unlikely that it is allowed to learn the predicates, making a strong case for the requirement of both plaintext privacy and predicate privacy.

The functionality of our construction is developed with the applications in the critical infrastructure (CI) domain in mind. The benefits of information sharing are widely acknowledged PCC97, but stakeholders still very reluctant in sharing their information with other parties SSF16, DS09, MS02]. We give two concrete use cases.

- Detection of coordinated attacks. While a single failure of a system in a CI may occur occasionally, a sudden failure of multiple systems from distinct CI operators, could be an indication of a large scale cyberattack. By centrally monitoring the "failure"/"running" status messages of the CI operators, a warning can be given to the national computer emergency response team whenever a combination of systems fails, allowing further investigation of the failures. Additionally, instead of sharing just binary messages to indicate whether a system has failed, it is also helpful to share and monitor cyberalert levels. These cyberalert levels from different clients are used to get an improved situational overview [LK15].

- Monitoring of dependencies among CI operators. There exist many dependencies among various CIs $\left[\mathrm{LNK}^{+} 09\right]$, making it possible for disruptions to easily propagate from one infrastructure to another $\mathrm{CLO}^{+} 06$. By timely reporting status messages on supply, a central authority can determine whether supply will meet demand and otherwise instruct parties to prepare their backup resources. Similarly, the sharing of compliance status (e.g., whether they can be met or not) can be used to take the right security measures at another party LK15.

\subsection{Related Work}

A multi-input functional encryption (MI-FE) $\mathrm{GGG}^{+} 14$ scheme is an FE scheme that supports the computation of functions over multiple encrypted inputs. Examples of special-purpose MI-FE include property-preserving encryption PR12, such as for ordering $\left.\mathrm{BLR}^{+} 15 . \mathrm{CLW}^{+} 16\right]$ or equality $\left[\mathrm{YTH}^{+} 10\right]$, and multi-input inner product encryption (MI-IPE) $\left[\mathrm{AGR}^{+} 17\right]$. The MI-IPE scheme by Abdalla et al. $\mathrm{AGR}^{+} 17$ is capable of computing the inner product of two vectors, i.e., the decryption algorithm returns a scalar. This should not be confused with an inner-product predicate encryption scheme where predicates (with a TRUE/

single message, e.g., $f\left(x_{1}, \ldots, x_{n}\right)=$ TRUE if and only if $x_{1}=0$. 
FALSE result) can be evaluated by an inner product. A private-key, multi-client $\mathrm{FE}(\mathrm{MC}-\mathrm{FE})$ scheme $\mathrm{GKL}^{+} 13 \mathrm{GGG}^{+} 14$ is a variant of MI-FE. There are two key differences between the two notions. Firstly, MC-FE requires that the ciphertexts for the function inputs are generated by individual distinct parties, while in MIFE it is allowed to have only a single encryptor for all the inputs. Secondly, in MC-FE the ciphertexts are associated with a time-step $\mathrm{GGG}^{+} 14$ or identifier. Such an identifier is used to prevent mix-and-match attacks: decryption only works when all ciphertexts are associated with the same identifier.

Although not recognized as such, several special-purpose MC-FE schemes have already been proposed in literature. Shi et al. $\mathrm{SCR}^{+} 11$ propose a construction for the privacy-preserving aggregation of time-series data. Their construction allows a central party to compute and learn the sum over encrypted numbers, without learning the individual numbers themselves. Decentralized multi-authority attribute-based encryption (MA-ABE) [LW11] can also be considered a form of MC-FE. In MA-ABE, several decryption keys, issued by different authorities and associated with an identifier, need to be combined to decrypt a single ciphertext. The similarity becomes apparent once we swap the roles of the ciphertext and decryption keys.

Wildcards have been used in PE before by Abdalla et al. $\mathrm{ACD}^{+} 06$ in IBE and by Boneh and Waters BW07 in HVE. These works differ from our work in several aspects. Most importantly, our construction is a multi-client variant instead of single-client. If we would apply a single-client construction in a multi-client setting, we would leak the individual predicate results for each party. Secondly, we achieve both plaintext privacy and predicate privacy, which is known to be impossible to accomplish in the public-key setting [SSW09] $\left(\left[\mathrm{ACD}^{+} 06, \mathrm{BW} 07\right.\right.$ are in the public-key setting). Finally, we look at predicate-only encryption, not at regular PE in which the ciphertexts may also contain an encrypted payload message.

Numerous PE schemes are used for searchable encryption (SE) $\mathrm{BHJ}^{+} 14$. However, we see no great benefit in applying MC-PoE as an SE scheme. MC-POE enables us to compute a predicate over multiple inputs from several explicitly chosen clients. In SE, this would correspond to a search over documents where the query specifies which keywords have to be set by which parties. This is also the reason why existing multi-writer $\left[\mathrm{BHJ}^{+} 14\right]$ schemes, do not consider searching over documents using queries which, for example, specify that party $p_{1}$ should have added keyword $w_{1}$, while party $p_{2}$ should have added keyword $w_{2}$. 


\section{Preliminaries}

Throughout this paper, we use $x \stackrel{R}{\leftarrow} S$ to denote that $x$ is chosen uniformly at random from the finite set $S$. We denote the $i$ th component of a vector $\boldsymbol{v}$ as $v_{i}$. For a set of indices $I$, we write $\boldsymbol{v}_{I}$ for the subvector of $\boldsymbol{v}$. Instead of consistently using the vector notation, we use set notation when this is more convenient.

\subsection{Primitives and Assumptions}

Our construction uses asymmetric bilinear maps.

Definition 1 (Bilinear Map). Let $\mathbb{G}_{1}, \mathbb{G}_{2}$, and $\mathbb{G}_{T}$ be cyclic multiplicative groups of prime order $p$. The map $e: \mathbb{G}_{1} \times \mathbb{G}_{2} \rightarrow \mathbb{G}_{T}$ is an asymmetric bilinear map if the following two conditions hold.

- The map is bilinear; $\forall g_{1} \in \mathbb{G}_{1}, g_{2} \in \mathbb{G}_{2}, a, b \in \mathbb{Z}_{p}: e\left(g_{1}^{a}, g_{2}^{b}\right)=e\left(g_{1}, g_{2}\right)^{a b}$.

- The map is non-degenerate; generators $g_{1}$ and $g_{2}$ are chosen such that the order of the element $e\left(g_{1}, g_{2}\right) \in \mathbb{G}_{T}$ equals $p$, the order of group $\mathbb{G}_{T}$.

More specifically, we use a Type 3 pairing [GPS08], where no efficiently computable homomorphisms between the groups $\mathbb{G}_{1}$ and $\mathbb{G}_{2}$ can be found.

We use the function $\mathcal{G}\left(1^{\kappa}\right)$ to generate the parameters for a Type 3 bilinear group for the security parameter $\kappa$.

Additionally, we use a pseudorandom permutation (PRP) over $\mathcal{M} \subseteq \mathbb{Z}_{p}$.

Definition 2 (Pseudorandom Function). For key space $\mathcal{K}$ and message space $\mathcal{M}$ define the function $\pi: \mathcal{K} \times \mathcal{M} \rightarrow \mathcal{M}$. The function $\pi$ is a pseudorandom permutation (PRP) if the output of $\pi$ is indistinguishable from the output of a permutation chosen uniformly at random from the set of all possible permutations over $\mathcal{M}$.

The security of our construction is based on the decisional Diffie-Hellman (DDH) problem and the symmetric external Diffie-Hellman (SXDH) problem.

Assumption 1. The decisional Diffie-Hellman (DDH) assumption states that, given $\left(\mathbb{G}, g \in \mathbb{G}, g^{a}, g^{b}, Z\right)$ for uniformly at random chosen $a$ and $b$, it is hard to distinguish $Z=g^{a b}$ from $Z \stackrel{R}{\leftarrow} \mathbb{G}$.

Assumption 2. Given the bilinear groups $\mathbb{G}_{1}$ and $\mathbb{G}_{2}$, the symmetric external Diffie-Hellman (SXDH) assumption states that the DDH problem in both group $\mathbb{G}_{1}$ and group $\mathbb{G}_{2}$ is hard. 


\section{Multi-client Predicate-only Encryption}

A multi-client predicate-only encryption scheme is a collection of the following four polynomial-time algorithms.

Setup $\left(1^{\kappa}, n\right)$. This algorithm defines the public parameters $\mathrm{pp}$, a master secret key msk, and the encryption keys usk ${ }_{i}$ for every client $1 \leq i \leq n$. The algorithm also defines the finite message space $\mathcal{M}^{n}$ and the predicate family $\mathcal{F}$, which predicates are efficiently computable on $\mathcal{M}^{n}$.

Encrypt(usk ${ }_{i}$, id, $x_{i}$ ). A client $i$ can encrypt a value $x_{i} \in \mathcal{M}$ using its encryption key usk $_{i}$ and an identifier id. Different clients can use the same identifier, however, each client can only use an identifier at most once. The algorithm returns a ciphertext $\mathrm{ct}_{\mathrm{id}, i}$. We usually omit the index id when there is no ambiguity. Furthermore, we introduce the following simplification of notation for a set of ciphertexts associated with the same id: For an ordered set $S \subseteq\{1, \ldots, n\}$ of indices, we write the set of ciphertexts $\left\{\right.$ Encrypt(usk $_{j}$, id, $\left.\left.x_{j}\right) \mid j \in S\right\}$ as Encrypt(usk ${ }_{S}$, id, $\boldsymbol{x}_{S}$ ). If $S=\{1, \ldots, n\}$, we simply write Encrypt(usk, id, $\boldsymbol{x}$ ) or $\mathrm{Ct}_{\boldsymbol{x}}$.

GenToken(msk, $f$ ). The key generator can create a decryption key, termed token, for predicate $f \in \mathcal{F}$ using the msk. The algorithm returns the token $\mathrm{tk}_{f}$.

$\operatorname{Test}\left(\mathrm{tk}_{f}, \mathrm{ct}_{\boldsymbol{x}}\right)$. The Test algorithm requires a vector of ciphertexts $\mathrm{ct}_{\boldsymbol{x}}$ and a token $\mathrm{tk}_{f}$ as input. The algorithm outputs a Boolean value.

Definition 3 (Correctness). A multi-client predicate-only encryption scheme is correct if $\operatorname{Test}\left(\mathrm{tk}_{f}, \mathrm{ct}_{\boldsymbol{x}}\right)=f(\boldsymbol{x})$. Formally, we require for all $n \in \mathbb{N}, \boldsymbol{x} \in \mathcal{M}^{n}$, and $f \in \mathcal{F}$,

$$
\operatorname{Pr}\left[\begin{array}{rl}
\left(\mathrm{pp}, \mathrm{msk},\left\{\text { usk }_{i}\right\}\right) \leftarrow \operatorname{Setup}\left(1^{\kappa}, n\right) \\
\operatorname{Test}_{\boldsymbol{x}} & \leftarrow \operatorname{Encrypt}(\text { usk, id, } \boldsymbol{x}) \\
\left.\mathrm{tk}_{f}, \mathrm{tk}_{f}\right) \neq f(\boldsymbol{x}): \operatorname{GenToken}(\mathrm{msk}, f)
\end{array}\right]
$$

is negligible in the security parameter $\kappa$, where the probability is taken over the coins of Setup, Encrypt, and GenToken.

Note that we do not impose any restriction on the output of Test if it operates on messages encrypted under different identifiers.

\subsection{Security}

A commonly considered security game for private-key functional encryption is an indistinguishability-based notion under which the adversary may query both 
the Encrypt and the GenToken oracles KSW08, SSW09, $\left.\mathrm{GGG}^{+} 14\right]$. Since our MC-PoE is a special case of MC-FE, we start from the security notion from Goldwasser et al. $\mathrm{GGG}^{+} 14$. However, they only consider the indistinguishability of plaintexts (plaintext privacy [KSW08, SSW09]) and not of functions (function or predicate privacy [BS15, SSW09]) in their security definition. In the following full security notion, we combine the plaintext-privacy and predicate-privacy notions, similarly to Shen et al. [SSW09].

Because an evaluation of a predicate on a set of messages reveals some information about the messages in relation to the predicate (and vice versa), we cannot allow the adversary to query for all combinations of messages and predicates. For example, an adversary can distinguish an encryption of message $\boldsymbol{x}_{0}$ from an encryption of $\boldsymbol{x}_{1}$ if it has a token for a predicate $f$ such that $f\left(\boldsymbol{x}_{0}\right) \neq f\left(\boldsymbol{x}_{1}\right)$. Even if we require $f\left(\boldsymbol{x}_{0}\right)=f\left(\boldsymbol{x}_{1}\right)$ for all predicates $f$ that the adversary queried, a similar situation can still appear. To see this, consider an adversary corrupting client $i$ so that it can encrypt any message $m_{i}$ as $i$ th input. This means that the adversary can also trivially distinguish the two messages if there exists a value $m_{i}$, such that if it replaces the $i$ th input of $\boldsymbol{x}_{0}$ and $\boldsymbol{x}_{1}$ by $m_{i}$ (resulting in inputs $\boldsymbol{x}_{0}^{\prime}$ and $\boldsymbol{x}_{1}^{\prime}$ respectively), the predicate has different outputs, i.e., $f\left(\boldsymbol{x}_{0}^{\prime}\right) \neq f\left(\boldsymbol{x}_{1}^{\prime}\right)$. Likewise, we also have to require that the predicates $f_{0}$ and $f_{1}$ yield the same result on a queried input $\boldsymbol{x}$, even if the adversary replaces some of the corrupted clients' inputs by another value.

In our security definition, we use the term static corruptions to indicate that the adversary announces the corrupted clients at the beginning of the game and cannot corrupt additional clients during the rest of the game. We let $I$ be the set of indices of the uncorrupted clients and, similarly, indicate the indices of the corrupted clients by the set $\bar{I}$. Recall that we use the notation $\boldsymbol{x}_{I}$ to denote the subvector of $\boldsymbol{x}$ containing only the components from the set $I$. We denote with $f\left(\boldsymbol{x}_{I}, \cdot\right)$ a predicate $f$ with the pre-filled inputs $\boldsymbol{x}_{I}$.

Definition 4 (Full Security). A multi-client predicate-only encryption scheme is adaptive full secure under static corruptions if every probabilistic polynomial time adversary $\mathcal{A}$ has at most a negligible advantage in winning the following game.

Initialization The adversary $\mathcal{A}$ submits a set of indices $\bar{I}$ to the challenger. We define the complement set $I=\{1, \ldots, n\} \backslash \bar{I}$.

Setup The challenger runs $\operatorname{Setup}\left(1^{\kappa}, n\right)$ to get the pp, msk, and $\left\{\text { usk }_{i}\right\}_{1 \leq i \leq n}$. It gives the public parameters pp and corrupted clients' keys $\left\{\right.$ usk $\left._{i} \mid i \in \bar{I}\right\}$ to the adversary.

Query 1 The adversary $\mathcal{A}$ may query the challenger for ciphertexts or tokens. 
- In case of a ciphertext query for $\left(i\right.$, id,$\left.x_{i}\right)$, the challenger returns $\mathrm{ct}_{\mathrm{id}, i} \leftarrow$ Encrypt(usk ${ }_{i}$, id, $\left.x_{i}\right)$.

- For a token query for $f$, the challenger returns $\mathrm{tk}_{f} \leftarrow$ GenToken(msk, $f$ ).

Challenge The challenger picks a random bit $b$. The adversary can either request a ciphertext challenge or a token challenge.

- In case of a ciphertext challenge, the adversary sends $\left(\mathrm{id}^{*}, \boldsymbol{x}_{0, I}^{*}, \boldsymbol{x}_{1, I}^{*}\right)$ to the challenger and receives the challenge $\mathrm{Ch}_{I} \leftarrow$ Encrypt $\left(\right.$ usk $\left._{I}, \mathrm{id}^{*}, \boldsymbol{x}_{b, I}^{*}\right)$ in return.

- In case of a token challenge, the adversary sends $\left(f_{0}^{*}, f_{1}^{*}\right)$ to the challenger. The challenger returns the challenge $\mathrm{Ch} \leftarrow \operatorname{GenToken}\left(\mathrm{msk}, f_{b}^{*}\right)$.

Query 2 The adversary may query the challenger again, similar to Query 1.

Guess The adversary outputs its guess $b^{\prime} \in\{0,1\}$ for the bit $b$.

We say that adversary $\mathcal{A}$ wins the game, if $b^{\prime}=b$ and

- in case of a ciphertext challenge, $\mathcal{A}$ did not query for a ciphertext using identifier id* in any of the two query phases, nor query for a predicate $f$, such that $f\left(\boldsymbol{x}_{0, I}^{*}, \cdot\right) \neq f\left(\boldsymbol{x}_{1, I}^{*}, \cdot\right)$;

- in case of a token challenge, $\mathcal{A}$ did not query for $\left(i\right.$, id, $\left.x_{i}\right)$, for uncorrupted clients $i \in I$, such that it can combine these inputs $x_{i}$ for the same id, into a vector $\boldsymbol{x}_{I}$, where $f_{0}^{*}\left(\boldsymbol{x}_{I}, \cdot\right) \neq f_{1}^{*}\left(\boldsymbol{x}_{I}, \cdot\right)$.

Note that in the above defined game, in case of a ciphertext challenge, the challenger only returns challenge ciphertexts for the uncorrupted clients. The adversary can still evaluate predicates on the received challenge by generating the ciphertext values for the corrupted clients using their encryption keys.

It is important to realize that the challenger can decide whether the adversary wins the game or not in polynomial time. This is possible because the adversary $\mathcal{A}$ can only query for a polynomial number of ciphertexts and tokens. Moreover, the challenger is able to efficiently check if $f\left(\boldsymbol{x}_{I}, \cdot\right)=f^{\prime}\left(\boldsymbol{x}_{I}^{\prime}, \cdot\right)$ as both $n$ and $\mathcal{M}^{n}$ are finite and fixed by $\operatorname{Setup}\left(1^{\kappa}, n\right)$.

Definition 5 (Selective Full Security). The definition of a selective full secure under static corruptions multi-client predicate-only encryption scheme is similar to the adaptive full security notion of Definition 4 The difference between the two, is that in selective security game, the challenge request (i.e., either $\left(\mathrm{id}^{*}, \boldsymbol{x}_{0, I}^{*}, \boldsymbol{x}_{1, I}^{*}\right)$ or $\left.\left(f_{0}^{*}, f_{1}^{*}\right)\right)$ is announced during Initialization. 
As explained before, the full security definition actually defines two security notions. We say that an MC-PoE scheme is adaptive (selective) plaintext private if no adversary can win the adaptive (selective, respectively) full security game with a ciphertext challenge. Similarly, an MC-PoE scheme is adaptive (selective) predicate private if no adversary can win the adaptive (selective, respectively) full security game with a token challenge.

Chosen-Plaintext Security. The definition of full security is very strong as it allows an adversary to query for both ciphertexts and tokens. This is similar to the chosen-ciphertext attack ( $\mathrm{CCA}$ ) security notion used in public-key cryptography, where the adversary can query both the encryption and decryption ${ }^{2}$ oracle. To accommodate for a different attacker model, we define a chosen-plaintext security notion, where the adversary only has access to the encryption oracle and is asked to distinguish between two ciphertexts. Such a notion is similar to chosen-plaintext attack (CPA) security as defined in public-key cryptography and is also related to the offline security notion of Lewi and Wu LW16], in which an attacker has only access to ciphertexts and not to decryption keys. To make our notion stronger, we give the adversary access to all clients' encryption keys (but not to the internal randomness of the clients).

Definition 6 (Chosen-Plaintext Security). A multi-client predicate-only encryption scheme is chosen-plaintext secure under unbounded corruptions if any probabilistic polynomial time algorithm $\mathcal{A}$ has at most a negligible advantage in winning the following game.

Setup The challenger runs Setup $\left(1^{\kappa}, n\right)$ to get the pp, msk, and $\left\{\text { usk }_{i}\right\}_{1 \leq i \leq n}$. It gives the public parameters pp and all clients' keys $\left\{\text { usk }_{i}\right\}_{1 \leq i \leq n}$ to the adversary. Note that the adversary $\mathcal{A}$ can encrypt any message $x_{i}$ for identifier id using the key usk $_{i}$ by computing Encrypt(usk ${ }_{i}$, id,$x_{i}$ ).

Challenge The adversary sends the challenge request $\left(\mathrm{id}^{*}, \boldsymbol{x}_{0}^{*}, \boldsymbol{x}_{1}^{*}\right)$ to the challenger. The challenger picks a random bit $b$ and returns Encrypt(usk, id ${ }^{*}, \boldsymbol{x}_{b}^{*}$ ) to the adversary.

Guess The adversary outputs its guess $b^{\prime} \in\{0,1\}$ for the bit $b$.

We say that adversary $\mathcal{A}$ wins the game if $b^{\prime}=b$.

Observe that in this game the adversary is given every client's private key. This security requirement is quite strong and corresponds to a following situation: Even if an attacker compromises a client and steals its encryption keys,

\footnotetext{
${ }^{2}$ In MC-PoE, an adversary can use a token and the public Test algorithm to learn more about the encrypted plaintext.
} 


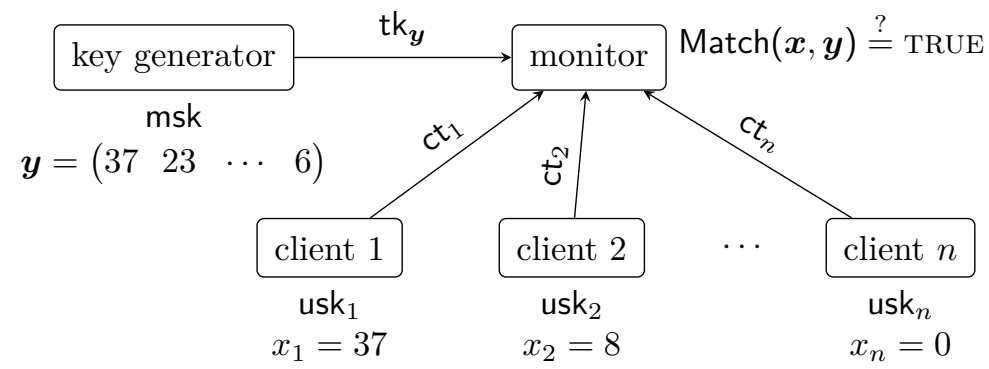

Figure 1: In this example of a multi-client monitoring system, there are $n$ distinct clients (with keys usk $_{1}, \ldots$, usk $_{n}$ ) that determine the values $x_{1}, \ldots, x_{n}$. The monitor computes the functionality $\operatorname{Match}(\boldsymbol{x}, \boldsymbol{y})$ using the encrypted values $\mathrm{ct}_{1}, \ldots, \mathrm{ct}_{n}$ and a token $\mathrm{tk}_{\boldsymbol{y}}$. The monitor is only able to compute the functionality if all clients encrypted their value $x_{i}$ using the same identifier id (not shown in the figure).

it remains hard for the attacker to determine the plaintexts of the ciphertexts created before and after the compromise.

\section{Our Construction}

We construct a multi-client predicate-only encryption scheme for the functionality of a conjunctive equality test. To test if $n$ messages $x_{1}, \ldots, x_{n}$, encrypted by distinct clients, equal the values $y_{1}, \ldots, y_{n}$, we evaluate the predicate

$$
\operatorname{Match}(\boldsymbol{x}, \boldsymbol{y})= \begin{cases}\text { TRUE } & \text { if } \bigwedge_{i=1}^{n}\left(x_{i}=y_{i}\right), \\ \text { FALSE } & \text { otherwise }\end{cases}
$$

As discussed in Section 1.1 this functionality turns out to be surprisingly useful in the domain of critical infrastructure protection. In this setting, a monitor combines the ciphertexts associated with the same identifier and evaluates all its tokens (corresponding to various predicates) on the ciphertext vector to see if there is a match. If a match is found, the monitor may raise an alarm or take other appropriate actions. A schematic overview of relations among all parties of such a multi-client monitoring system is shown in Figure 1

We now describe our multi-client predicate-only encryption construction for conjunctive equality tests over multiple clients.

$\operatorname{Setup}\left(1^{\kappa}, n\right)$. Let $\left(p, \mathbb{G}_{1}, \mathbb{G}_{2}, \mathbb{G}_{T}, e, g_{1}, g_{2}\right) \leftarrow \mathcal{G}\left(1^{\kappa}\right)$ be the parameters for a bilinear group. Choose a pseudorandom permutation $\pi: \mathcal{K} \times \mathcal{M} \rightarrow \mathcal{M}$ for message 
space $\mathcal{M} \subseteq \mathbb{Z}_{p}$ and a cryptographic hash function $H:\{0,1\}^{*} \rightarrow \mathbb{G}_{1}$. The bilinear group parameters together with both functions form the public parameters. To generate the keys, select $\alpha_{i}, \gamma_{i} \stackrel{R}{\leftarrow} \mathbb{Z}_{p}^{*}$ and $\beta_{i} \stackrel{R}{\leftarrow} \mathcal{K}$ for $1 \leq i \leq n$. The master secret key is

$$
\text { msk }=\left\{\left(g_{2}^{\alpha_{i}}, \beta_{i}, g_{2}^{\gamma_{i}}\right)\right\}_{i=1}^{n} .
$$

The secret encryption key for client $i$ is

$$
\mathrm{usk}_{i}=\left(g_{1}^{\alpha_{i}}, \beta_{i}, \gamma_{i}\right)
$$

Encrypt(usk ${ }_{i}$, id, $x_{i}$ ). Client $i$ can encrypt its message $x_{i} \in \mathcal{M}$ for identifier id using usk $_{i}$ and $r_{i} \stackrel{R}{\leftarrow} \mathbb{Z}_{p}^{*}$,

$$
\mathrm{ct}_{i}=\left(H(\mathrm{id}), g_{1}^{r_{i}}, g_{1}^{\alpha_{i} \pi\left(\beta_{i}, x_{i}\right) r_{i}} H(\mathrm{id})^{\gamma_{i}}\right) .
$$

Gen Token(msk, $\boldsymbol{y})$. The token generator can encrypt a vector $\boldsymbol{y} \in \mathcal{M}^{n}$ using its key msk. Choose $u_{i} \stackrel{R}{\leftarrow} \mathbb{Z}_{p}^{*}$ for $1 \leq i \leq n$ and output

$$
\mathrm{tk}_{\boldsymbol{y}}=\left(\left\{g_{2}^{u_{i}}, g_{2}^{\alpha_{i} \pi\left(\beta_{i}, y_{i}\right) u_{i}} \mid 1 \leq i \leq n\right\}, \prod_{1 \leq i \leq n}\left(g_{2}^{\gamma_{i}}\right)^{u_{i}}\right)
$$

Test $\left(\mathrm{tk}_{\boldsymbol{y}},\left\{\mathrm{ct}_{i}\right\}_{1 \leq i \leq n}\right)$. Output the result of the test

$$
\begin{aligned}
\prod_{1 \leq i \leq n} e\left(g_{1}^{\alpha_{i} \pi\left(\beta_{i}, x_{i}\right) r_{i}} H(\mathrm{id})^{\gamma_{i}}, g_{2}^{u_{i}}\right) \stackrel{?}{=} \\
\\
\prod_{1 \leq i \leq n} e\left(g_{1}^{r_{i}}, g_{2}^{\alpha_{i} \pi\left(\beta_{i}, y_{i}\right) u_{i}}\right) e\left(H(\mathrm{id}), \prod_{1 \leq i \leq n}\left(g_{2}^{\gamma_{i}}\right)^{u_{i}}\right) .
\end{aligned}
$$

\subsection{Correctness}

Correctness follows from the definition of Test. We remark that the output of Test is completely determined by $\sum_{1 \leq i \leq n}\left(\pi\left(\beta_{i}, x_{i}\right)-\pi\left(\beta_{i}, y_{i}\right)\right) \stackrel{?}{=} 0$. Since the function $\pi$ is a PRP, the probability of Test $\left(\operatorname{tk}_{\boldsymbol{y}}, \mathrm{ct}_{\boldsymbol{x}}\right) \neq \operatorname{Match}(\boldsymbol{x}, \boldsymbol{y})$ is negligible.

\subsection{Security}

To get an intuition for the security of our construction, observe that the clients' messages itself are first encrypted using the PRP $\pi$. By using the output of the 
PRP as an exponent and randomizing it with the value $r$, we create a probabilistic encryption of the message. The PRP's randomized output also prevents malleability attacks. Similarly, the vector components of the vector $\boldsymbol{y}$ are individually encrypted in a similar way. Because part of the clients' keys (i.e., $g_{1}^{\alpha_{i}}$ ) and the master secret key $\left(i . e ., g_{2}^{\alpha_{i}}\right)$ reside in different groups, it is hard for a client to create a token and hard for the token generator to create a ciphertext.

The formal security analysis can be found in Appendix A. We prove our construction selective plaintext private and adaptive predicate private. Additionally, we prove the chosen-plaintext security property of the construction. Plaintext and predicate privacy are proven in the generic group model using random oracles. This combination of models has been successfully applied in other works before [CMZ14 Sma01]. Chosen-plaintext security can be proven in the standard model and under the DDH assumption in group $\mathbb{G}_{1}$. We formulate the following two theorems.

Theorem 1. Let $\mathcal{A}$ be an arbitrary probabilistic polynomial time adversary having oracle access to the group operations and the encryption and token generation algorithms, while it is bounded in receiving at most $q$ distinct group elements. The adversary $\mathcal{A}$ has at most an advantage of $O\left(q^{2} / p\right)$ in winning either the selective plaintext-privacy (see Definition 5) or the adaptive predicate-privacy game (see Definition 4) in the random oracle model.

Theorem 2. The construction presented above is chosen-plaintext secure with an unbounded number of corruptions (Definition 6) under the DDH assumption in group $\mathbb{G}_{1}$.

Both plaintext privacy and predicate privacy are proven secure through a series of hybrid games. In every game hop, a component of the challenge vector (either the ciphertext or token challenge vector) is replaced by a random one. In the final game, once all components are replaced by random elements, no adversary can gain an advantage since it is impossible to distinguish a random vector from another random one.

However, in the selective plaintext-privacy game, not every component of the challenge vector can be replaced by a random component. If a component $x_{b, i}^{*}$ of the challenge vector $\boldsymbol{x}_{b}^{*}$ is deterministic, i.e., the challenge inputs were the same for that component, $x_{0, i}^{*}=x_{1, i}^{*}=m$, the adversary may query for a token to match this single component for the value $y_{i}=m$. Note that if this component is replaced by a random element, Match will, with overwhelming probability, return FALSE, while it should have returned TRUE. Hence, the deterministic components of the challenge vector have to remain untouched in every game hop. This implies that the number of game hops depends on the challenge inputs, requiring the challenger to know the challenge inputs a priori. This 
limitation does not appear for predicate privacy, making it possible to prove adaptive security instead.

\subsection{Extension Allowing Wildcards}

Although a construction for the described conjunctive equality matching functionality would suffice, it may be very inefficient when a predicate is defined over a subset of the clients' inputs. For example, suppose the token generator has a predicate for which it actually does not care what client $i$ sends. Now, if we have only conjunctive equality matching, we would need to create a token for every possible message that client $i$ can send. Besides that this will be very inefficient if client $i$ could send many different messages, it would also reveal whenever client $i$ has sent the same values multiple times: whenever a client sends the same value multiple times, the same token will match multiple times as well!

We can extend our construction with the ability to test for the equality of vectors with the additional feature that the predicate vector $\boldsymbol{y}$ can now contain wildcard components. Such a wildcard component matches against any value of the corresponding ciphertext component. This makes the testing functionality similar to the one used in HVE [BW07], however our system combines the ciphertexts from multiple clients. Formally, the clients encrypt their messages from the message space $\mathcal{M} \subseteq \mathbb{Z}_{p}$, where the token generator uses the space $\mathcal{M}^{*}=\mathcal{M} \cup\{\star\}$. The multi-client predicate-only encryption construction now evaluates the function

$$
\operatorname{Match}^{\star}(\boldsymbol{x}, \boldsymbol{y})= \begin{cases}\text { TRUE } & \text { if } \forall i:\left(x_{i}=y_{i}\right) \vee\left(y_{i}=\star\right) \\ \text { FALSE } & \text { otherwise }\end{cases}
$$

To achieve this additional functionality, we have to change the GenToken and Test algorithms, the other algorithms remain unchanged.

Gen Token ${ }^{\star}($ msk, $\boldsymbol{y})$. The token generator can encrypt a predicate vector $\boldsymbol{y} \in$ $\left(\mathcal{M}^{*}\right)^{n}$ using the master secret key msk. Let $S_{\boldsymbol{y}}$ be the set of indices of the non-wildcard components of the vector $\boldsymbol{y}$. Choose $u_{i} \stackrel{R}{\leftarrow} \mathbb{Z}_{p}^{*}$ for $i \in S_{\boldsymbol{y}}$ and output

$$
\mathrm{tk}_{\boldsymbol{y}}=\left(\left\{g_{2}^{u_{i}}, g_{2}^{\alpha_{i} \pi\left(\beta_{i}, y_{i}\right) u_{i}} \mid i \in S_{\boldsymbol{y}}\right\}, \prod_{i \in S_{\boldsymbol{y}}}\left(g_{2}^{\gamma_{i}}\right)^{u_{i}}\right)
$$


Test $^{\star}\left(\mathrm{tk}_{\boldsymbol{y}},\left\{\mathrm{ct}_{i}\right\}_{i \in S_{y}}\right)$. Output the result of the test

$$
\begin{aligned}
\prod_{i \in S_{y}} e\left(g_{1}^{\alpha_{i} \pi\left(\beta_{i}, x_{i}\right) r_{i}} H(\mathrm{id})^{\gamma_{i}}, g_{2}^{u_{i}}\right) \stackrel{?}{=} \\
\prod_{i \in S_{y}} e\left(g_{1}^{r_{i}}, g_{2}^{\alpha_{i} \pi\left(\beta_{i}, y_{i}\right) u_{i}}\right) e\left(H(\mathrm{id}), \prod_{i \in S_{y}}\left(g_{2}^{\gamma_{i}}\right)^{u_{i}}\right) .
\end{aligned}
$$

In this adapted construction, the wildcards are made possible by allowing the token generator to specify which clients need to contribute a ciphertext before one can evaluate the predicate over the subset of clients. This idea is encoded in the token by the value $\prod_{i \in S_{y}}\left(g_{2}^{\gamma_{i}}\right)^{u_{i}}$ and in the ciphertext by the value $H(\text { id })^{\gamma_{i}}$. The latter also prevents the monitor to combine ciphertext for different identifiers.

The addition of wildcards to the scheme should be mainly considered an efficiency improvement, rather than a security improvement, although the ciphertext security actually slightly improves when one uses wildcards - the wildcard components do not leak any information about the matched ciphertext, as discussed above. However, we point out that this adapted construction is not predicate private. In fact, if wildcards are used in the proposed construction, the token would leak their positions: by looking at a token, it is possible to tell which components encode a wildcard. But, if we accept this fact, yet still want to assure that no other information is leaked, we can define a restricted predicate-privacy game. In this restricted game, we restrict the adversary to only provide challenge inputs with wildcards in the same position, i.e., we require for challenge inputs $f_{0}^{*}=\boldsymbol{y}_{0}^{*}, f_{1}^{*}=\boldsymbol{y}_{1}^{*}$ that for all $1 \leq i \leq n, y_{0, i}=\star \Longleftrightarrow y_{1, i}=\star$.

It is trivial to see that changing the GenToken or Test algorithm does not influence the chosen-ciphertext security. In Appendix $\mathrm{A}$ we give the security proofs for the construction with wildcards.

\subsection{Efficiency}

Since the Encrypt and GenToken algorithms do not use any expensive pairing operations, they can efficiently run on less powerful hardware. For the Encrypt algorithm it is only needed to compute the PRP $\pi$ and three modular exponentiations. The computational complexity of GenToken` depends on the number of non-wildcard components in the predicate. For every non-wildcard component one evaluation of the PRP $\pi$ and three modular exponentiations are needed.

The Test algorithm is the only algorithm that requires pairings. To evaluate a token with $n$ non-wildcard components, $2 n+1$ pairing evaluations are needed.

In the next section we discuss a concrete implementation of the construction and evaluate its performance. 


\section{Implementation and Evaluation}

We have implemented a prototype of our construction with wildcards to get a better understanding of its performance. The implementation ${ }^{3}$ uses the PairingBased Cryptography Library that allows one to easily change the underlying curve and its parameters.

Instantiating the Pseudorandom Permutation. Our construction uses a PRP $\pi$ to permute an element in $\mathbb{Z}_{p}$. However, since we use the outcome of the permutation to exponentiate a generator in $\mathbb{G}_{1}$ and $\mathbb{G}_{2}$, we can instead directly map values in $\mathbb{Z}_{p}$ to one of these groups respectively. The pseudorandom function (PRF) proposed by Naor and Reingold [NR04] exactly achieves this. Their PRF maps a message $x \in \mathcal{M} \subseteq\left\{0, \ldots, 2^{m}-1\right\} \subseteq \mathbb{Z}_{p}$ using a key $\boldsymbol{b}=$ $\left\{b_{i} \stackrel{R}{\leftarrow} \mathbb{Z}_{p}^{*} \mid 0 \leq i \leq m\right\}$ to an element in a group $\langle g\rangle$ of prime order $p$. The PRF $F$ is defined as

$$
F(\boldsymbol{b}, x)=g^{b_{0} \prod_{i=1}^{m} b_{i}^{x[i]}},
$$

where $x[i] \in\{0,1\}$ denotes the $i$ th bit of message $x$. The advantage of using this PRF over a PRP is that it is relatively simple to compute while it is provably secure under the DDH assumption.

We apply the PRF to both the Encrypt and the GenToken` algorithms to obtain ciphertexts of the form

$$
\mathrm{ct}_{i}=\left(H(\mathrm{id}), g_{1}^{r_{i}}, g_{1}^{\alpha_{i} \prod_{j=1}^{m} \beta_{i, j}^{x_{i}[j]} r_{i}} H(\mathrm{id})^{\gamma_{i}}\right),
$$

and tokens of the form

$$
\mathrm{tk}_{\boldsymbol{y}}=\left(\left\{g_{2}^{u_{i}}, g_{2}^{\alpha_{i} \prod_{j=1}^{m} \beta_{i, j}^{y_{i}[j]} u_{i}} \mid i \in S_{\boldsymbol{y}}\right\}, \prod_{i \in S_{\boldsymbol{y}}}\left(g_{2}^{\gamma_{i}}\right)^{u_{i}}\right) .
$$

Notice that we use $b_{0}=\alpha_{i}$ and $b_{j}=\beta_{i, j}$. In addition, observe that it is not necessary to know the value $\alpha_{i}$ to compute a ciphertext or token, as long the value $g_{1}^{\alpha_{i}}$, or $g_{2}^{\alpha_{i}}$ respectively, is known.

Performance Measurements. We ran several performance evaluations on a notebook containing an Intel Core i5 CPU, running on Debian GNU/Linux. We

\footnotetext{
3 https://github.com/CRIPTIM/multi-client-monitoring

${ }^{4}$ https://crypto.stanford.edu/pbc/
} 


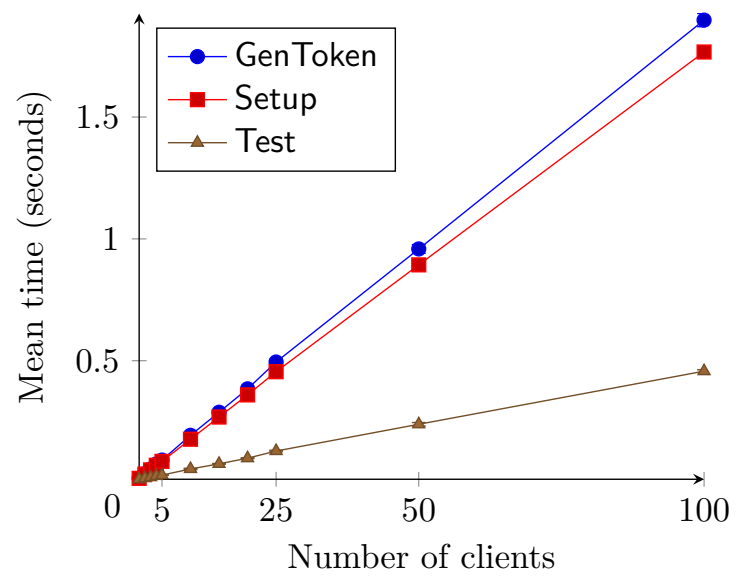

Figure 2: Performance measurements of the implementation using an MNT-159 curve.

chose to evaluate the system using an MNT curve MNT01 over a 159 bit base field size with embedding degree 6 .

As expected from the theoretical performance analysis in Section 4.4 both the GenToken ${ }^{\star}$ and Test ${ }^{\star}$ algorithms scale linearly in the number of non-wildcard components used. The GenToken ${ }^{\star}$ algorithm spends, on average, $19 \mathrm{~ms}$ to encrypt a non-wildcard component. To evaluate a token that contains no wildcards using $n$ ciphertexts, takes $4.5 n+10 \mathrm{~ms}$ on average. The Setup algorithm scales linearly as well, spending on average $18 \mathrm{~ms}$ per client to create their public and private keys. The Encrypt algorithm is the fastest, taking only $2.6 \mathrm{~ms}$ for an individual client to encrypt a message $x_{i} \in\{0, \ldots, 15\}$.

In Figure 2 the average computational time is plotted against the number of clients involved in the computation. No wildcards were used in the GenToken* and Test ${ }^{\star}$ algorithms to obtain these timing results, meaning that the algorithms are identical to GenToken and Test, respectively.

Considering an example of the monitoring of several CIs, we remark that a typical information-sharing community (e.g., an ISAC) consists of about 10 parties. So, if every party sends 5 distinct messages for each identifier (e.g., every party has five subsystems to be monitored), we would require a system of about 50 clients. We see that in such a realistically sized system we can evaluate about 250 predicates per minute. Optimizations such as the preprocessing of pairings can increase the number of predicate evaluations per minute. 


\section{Conclusion}

By designing a special-purpose multi-client functional encryption scheme, it is possible to create a practical privacy-preserving monitoring system. To achieve this, we defined multi-client predicate-only encryption (MC-POE) and corresponding security definitions for the protection of both the messages of the individual clients and the predicates. Our proposed construction for such an MC-PoE scheme is capable of conjunctive equality testing over vector components which can include wildcards. The performance evaluation of our implementation shows that the evaluation time of a predicate scales linearly in the number of clients, where a predicate defined over 20 clients can be evaluated in a tenth of a second. Additionally, we see that the encryption algorithm is very lightweight, making it suitable to run on resource-constrained devices.

Future work will include the construction of an MC-PoE scheme which will allow for more expressive functionality, while remaining efficient enough to run in practice and keeping the confidentiality of both the messages and the predicates. Additionally, further research is needed to construct an MC-PoE scheme that is fully secure in the standard model.

\section{Acknowledgments}

This work was supported by the Netherlands Organisation for Scientific Research (NWO) in the context of the CRIPTIM project. The authors additionally thank the reviewers and shepherd for their suggested improvements.

\section{References}

$\left[\mathrm{ACD}^{+}\right.$06] Michel Abdalla, Dario Catalano, Alexander W. Dent, John Malone-Lee, Gregory Neven, and Nigel P. Smart. "Identity-Based Encryption Gone Wild". In: International Colloquium on Automata, Languages, and Programming (ICALP). Ed. by Michele Bugliesi, Bart Preneel, Vladimiro Sassone, and Ingo Wegener. Vol. 4052.II. LNCS. Berlin, Heidelberg: Springer, 2006, pp. 300-311. ISBN: 978-3-540-35908-1. DOI: 10.1007/11787006_26

[AGR $\left.{ }^{+} 17\right]$ Michel Abdalla, Romain Gay, Mariana Raykova, and Hoeteck Wee. "Multi-Input Inner-Product Functional Encryption from Pairings". In: EUROCRYPT. Ed. by Jean-Sébastien Coron and Jesper Buus Nielsen. Vol. 10210.I. LNCS. Cham: Springer International Publishing, 2017, pp. 601-626. ISBN: 978-3-319-56620-7. DOI: 10.1007/978-3-319-566207_21. 
[BF01] Dan Boneh and Matt Franklin. "Identity-Based Encryption from the Weil Pairing". In: CRYPTO. Ed. by Joe Kilian. Vol. 2139. LNCS. Berlin, Heidelberg: Springer, 2001, pp. 213-229. ISBN: 978-3-540-44647-7. DOI: 10 . 1007/3-540-44647-8_13.

$\left[\mathrm{BHJ}^{+}{ }^{14}\right]$ Christoph Bösch, Pieter Hartel, Willem Jonker, and Andreas Peter. "A Survey of Provably Secure Searchable Encryption". In: ACM Computing Surveys (CSUR) 47.2 (Aug. 2014), 18:1-18:51. ISSN: 0360-0300. DOI: 10. $1145 / 2636328$.

[BLR $\left.{ }^{+} 15\right]$ Dan Boneh, Kevin Lewi, Mariana Raykova, Amit Sahai, Mark Zhandry, and Joe Zimmerman. "Semantically Secure Order-Revealing Encryption: Multi-input Functional Encryption Without Obfuscation". In: EUROCRYPT. Ed. by Elisabeth Oswald and Marc Fischlin. Vol. 9057.II. LNCS. Berlin, Heidelberg: Springer, 2015, pp. 563-594. ISBN: 978-3-662-46803-6. DOI: $10.1007 / 978-3-662-46803-6 \_19$.

[BS15] Zvika Brakerski and Gil Segev. "Function-Private Functional Encryption in the Private-Key Setting". In: Theory of Cryptography (TCC). Ed. by Yevgeniy Dodis and Jesper Buus Nielsen. Vol. 9015. LNCS. Berlin, Heidelberg: Springer, 2015, pp. 306-324. ISBN: 978-3-662-46497-7. DOI: 10 . 1007/978-3-662-46497-7_12

[BSW11] Dan Boneh, Amit Sahai, and Brent Waters. "Functional Encryption: Definitions and Challenges". In: Theory of Cryptography (TCC). Ed. by Yuval Ishai. Vol. 6597. LNCS. Berlin, Heidelberg: Springer, 2011, pp. 253-273. ISBN: 978-3-642-19570-9. DOI: 10.1007/978-3-642-19571-6_16

[BW07] Dan Boneh and Brent Waters. "Conjunctive, Subset, and Range Queries on Encrypted Data". In: Theory of Cryptography (TCC). Ed. by Salil P. Vadhan. Vol. 4392. LNCS. Berlin, Heidelberg: Springer, 2007, pp. 535554. ISBN: 978-3-540-70935-0. DOI: 10.1007/978-3-540-70936-7_29

$\left[\mathrm{CLO}^{+} 06\right] \quad$ S. H. Conrad, R. J. LeClaire, G. P. O'Reilly, and H. Uzunalioglu. "Critical national infrastructure reliability modeling and analysis". In: Bell Labs Technical Journal 11.3 (2006), pp. 57-71. ISSN: 1089-7089. DOI: 10.1002/ bltj.20178.

$\left[\mathrm{CLW}^{+} 16\right]$ Nathan Chenette, Kevin Lewi, Stephen A. Weis, and David J. Wu. "Practical Order-Revealing Encryption with Limited Leakage". In: Fast Software Encryption (FSE). Ed. by Thomas Peyrin. Vol. 9783. LNCS. Berlin, Heidelberg: Springer, 2016, pp. 474-493. ISBN: 978-3-662-52993-5. DOI: 10.1007/978-3-662-52993-5_24

[CMZ14] Melissa Chase, Sarah Meiklejohn, and Greg Zaverucha. "Algebraic MACs and Keyed-Verification Anonymous Credentials". In: Computer and Communications Security (CCS). New York, NY, USA: ACM, 2014, pp. 12051216. ISBN: 978-1-4503-2957-6. DOI: $10.1145 / 2660267.2660328$. 
[DS09] Myriam Dunn-Cavelty and Manuel Suter. "Public-Private Partnerships are no silver bullet: An expanded governance model for Critical Infrastructure Protection". In: International Journal of Critical Infrastructure Protection 2.4 (2009), pp. 179-187. ISSN: 1874-5482. DOI: $10.1016 / \mathrm{j}$. ijcip.2009.08.006

$\left[\mathrm{GGG}^{+} 14\right]$ Shafi Goldwasser, S. Dov Gordon, Vipul Goyal, Abhishek Jain, Jonathan Katz, Feng-Hao Liu, Amit Sahai, Elaine Shi, and Hong-Sheng Zhou. "Multi-input Functional Encryption". In: EUROCRYPT. Ed. by Phong Q. Nguyen and Elisabeth Oswald. Vol. 8441. LNCS. Berlin, Heidelberg: Springer, 2014, pp. 578-602. ISBN: 978-3-642-55219-9. DOI: 10.1007/9783-642-55220-5_32

$\left[\mathrm{GGH}^{+} 13\right] \quad$ S. Garg, C. Gentry, S. Halevi, M. Raykova, A. Sahai, and B. Waters. "Candidate Indistinguishability Obfuscation and Functional Encryption for all Circuits". In: Foundations of Computer Science (FOCS). 2013, pp. 40-49. DOI: 10.1109/FOCS.2013.13

$\left[\mathrm{GKL}^{+} 13\right]$ S. Dov Gordon, Jonathan Katz, Feng-Hao Liu, Elaine Shi, and HongSheng Zhou. Multi-Input Functional Encryption. Tech. rep. Nov. 2013. IACR: 2013/774

[GPS08] Steven D. Galbraith, Kenneth G. Paterson, and Nigel P. Smart. "Pairings for cryptographers". In: Discrete Applied Mathematics 156.16 (2008). Applications of Algebra to Cryptography, pp. 3113-3121. ISSN: 0166-218X. DOI: $10.1016 / \mathrm{j} . \mathrm{dam} .2007 .12 .010$

[KSW08] Jonathan Katz, Amit Sahai, and Brent Waters. "Predicate Encryption Supporting Disjunctions, Polynomial Equations, and Inner Products". In: EUROCRYPT. Ed. by Nigel Smart. Vol. 4965. LNCS. Berlin, Heidelberg: Springer, 2008, pp. 146-162. ISBN: 978-3-540-78967-3. DOI: 10.1007/9783-540-78967-3_9.

[LK15] Eric Luiijf and Marieke Klaver. "On the Sharing of Cyber Security Information". In: International Conference on Critical Infrastructure Protection (ICCIP). Ed. by Mason Rice and Sujeet Shenoi. Vol. 466. IFIP Advances in Information and Communication Technology (IFIPAICT). Cham, Heidelberg, New York, Dordrecht, London: Springer International Publishing, 2015, pp. 29-46. ISBN: 978-3-319-26567-4. DOI: 10.1007/9783-319-26567-4_3

[LNK $\left.{ }^{+} 09\right]$ Eric Luiijf, Albert Nieuwenhuijs, Marieke Klaver, Michel van Eeten, and Edite Cruz. "Empirical Findings on Critical Infrastructure Dependencies in Europe". In: Critical Information Infrastructure Security (CRITIS). Ed. by Roberto Setola and Stefan Geretshuber. Vol. 5508. LNCS. Berlin, Heidelberg: Springer, 2009, pp. 302-310. ISBN: 978-3-642-03552-4. DOI: 10 . 1007/978-3-642-03552-4_28 
[LW11] Allison Lewko and Brent Waters. "Decentralizing Attribute-Based Encryption". In: EUROCRYPT. Ed. by Kenneth G. Paterson. Vol. 6632. LNCS. Berlin, Heidelberg: Springer, 2011, pp. 568-588. ISBN: 978-3-64220464-7. DOI: 10.1007/978-3-642-20465-4_31

[LW16] Kevin Lewi and David J. Wu. "Order-Revealing Encryption: New Constructions, Applications, and Lower Bounds". In: Conference on Computer and Communications Security (CCS). New York, NY, USA: ACM, 2016. DOI: $10.1145 / 2976749.2978376$

[MNT01] Atsuko Miyaji, Masaki Nakabayashi, and Shunzo Takano. "Characterization of Elliptic Curve Traces Under FR-Reduction". In: International Conference on Information Security and Cryptology (ICISC). Ed. by Dongho Won. Vol. 2015. LNCS. Berlin, Heidelberg: Springer, 2001, pp. 90-108. ISBN: $978-3-540-45247-8$. DOI: $10.1007 / 3-540-45247-8 \_8$

[MS02] John D. Moteff and Gina M. Stevens. Critical Infrastructure Information Disclosure and Homeland Security. Report. Library of Congress Washington DC Congressional Research Service, Aug. 31, 2002. 23 pp. URL: http: //www.dtic.mil/docs/citations/ADA467310 (visited on 11/01/2017).

[NR04] Moni Naor and Omer Reingold. "Number-theoretic Constructions of Efficient Pseudo-random Functions". In: Journal of the ACM 51.2 (Mar. 2004), pp. 231-262. ISSN: 0004-5411. DOI: 10.1145/972639.972643

[ONe11] Adam O'Neill. Definitional Issues in Functional Encryption. Tech. rep. Mar. 2011. IACR: 2010/556

[PCC97] President's Commission on Critical Infrastructure Protection. Critical Foundations: Protecting America's Infrastructures. Report. Oct. 1997. URL: https : / / www . fas . org / sgp/library / pccip ·pdf (visited on 05/01/2016).

[PR12] Omkant Pandey and Yannis Rouselakis. "Property Preserving Symmetric Encryption". In: EUROCRYPT. Ed. by David Pointcheval and Thomas Johansson. Vol. 7237. LNCS. Berlin, Heidelberg: Springer, 2012, pp. 375391. ISBN: 978-3-642-29010-7. DOI: 10.1007/978-3-642-29011-4_23.

[Sch80] J. T. Schwartz. "Fast Probabilistic Algorithms for Verification of Polynomial Identities". In: Journal of the ACM 27.4 (Oct. 1980), pp. 701-717. ISSN: 0004-5411. DOI: $10.1145 / 322217.322225$.

[SCR $\left.{ }^{+} 11\right]$ Elaine Shi, T.-H. Hubert Chan, Eleanor G. Rieffel, Richard Chow, and Dawn Song. "Privacy-Preserving Aggregation of Time-Series Data". In: Network and Distributed System Security Symposium (NDSS). The Internet Society, 2011. URL: https://www .ndss-symposium .org/ndss2011/ privacy-preserving-aggregation-of-time-series-data/ (visited on $11 / 01 / 2017)$. 
[Sho97] Victor Shoup. "Lower Bounds for Discrete Logarithms and Related Problems". In: EUROCRYPT. Ed. by Walter Fumy. Vol. 1233. LNCS. Berlin, Heidelberg: Springer, 1997, pp. 256-266. ISBN: 978-3-540-69053-5. DOI: 10.1007/3-540-69053-0_18

[Sma01] N. P. Smart. "The Exact Security of ECIES in the Generic Group Model". In: IMA International Conference on Cryptography and Coding (IMACC). Ed. by Bahram Honary. Vol. 2260. LNCS. Berlin, Heidelberg: Springer, 2001, pp. 73-84. ISBN: 978-3-540-45325-3. DOI: $10.1007 / 3-540-45325-$ 3 3.8.

[SSF16] Florian Skopik, Giuseppe Settanni, and Roman Fiedler. "A problem shared is a problem halved: A survey on the dimensions of collective cyber defense through security information sharing". In: Computers $\&$ Security 60 (July 2016), pp. 154-176. ISSN: 0167-4048. DOI: $10.1016 / \mathrm{j}$. cose.2016.04.003.

[SSW09] Emily Shen, Elaine Shi, and Brent Waters. "Predicate Privacy in Encryption Systems". In: Theory of Cryptography (TCC). Ed. by Omer Reingold. Vol. 5444. LNCS. Berlin, Heidelberg: Springer, 2009, pp. 457-473. ISBN: 978-3-642-00456-8. DOI: 10.1007/978-3-642-00457-5_27

[SW05] Amit Sahai and Brent Waters. "Fuzzy Identity-Based Encryption". In: EUROCRYPT. Ed. by Ronald Cramer. Vol. 3494. LNCS. Berlin, Heidelberg: Springer, 2005, pp. 457-473. ISBN: 978-3-540-32055-5. DOI: 10.1007/ 11426639_27.

$\left[\mathrm{YTH}^{+} 10\right]$ Guomin Yang, Chik How Tan, Qiong Huang, and Duncan S. Wong. "Probabilistic Public Key Encryption with Equality Test". In: Cryptographers' Track at the RSA Conference (CT-RSA). Ed. by Josef Pieprzyk. Vol. 5985. LNCS. Berlin, Heidelberg: Springer, 2010, pp. 119-131. ISBN: 978-3-64211925-5. DOI: $10.1007 / 978-3-642-11925-5 \_9$

\section{A Security Proofs}

\section{A.1 Selective Plaintext and Adaptive Predicate Security}

We prove Theorem 1 stating that the construction without wildcards is secure, by using the following lemma and by proving that the construction with wildcards is selective plaintext private and restricted adaptive predicate private. Recall that the restricted predicate-private game is almost identical to our predicate-private game. However, in the restricted game, we additionally require $y_{0, i}^{*}=\star \Longleftrightarrow y_{1, i}^{*}=\star$ for the challenge inputs $\boldsymbol{y}_{0}^{*}, \boldsymbol{y}_{1}^{*}$.

Lemma 1. If the construction with wildcards is selective plaintext private and restricted adaptive predicate private, then the construction without wildcards is selective plaintext private and adaptive predicate private. 
Proof. First, let us look at the selective plaintext privacy. Assume $\mathcal{A}$ is a probabilistic polynomial time adversary, having a non-negligible advantage in winning the selective plaintext-privacy game without wildcards. It is clear that $\mathcal{A}$ is also an adversary that has an identical, non-negligible, advantage in winning the selective plaintext-privacy game with wildcards (however, it chooses not to use any). This contradicts with the given statement that no such adversary exists.

For the other part, assume that $\mathcal{A}$ is a probabilistic polynomial time adversary, making no wildcard queries, and having a non-negligible advantage in winning the predicate-privacy game. Note that $\mathcal{A}$ is also an adversary that has an identical, non-negligible, advantage in winning the predicate-privacy game with wildcards (however, it chooses not to use any). Specifically, since $\mathcal{A}$ chooses its challenge inputs without wildcards, $\mathcal{A}$ also satisfied the extra requirement in the restricted predicate-privacy game.

We now give a proof for both selective plaintext privacy as well as restricted predicate privacy for the construction with wildcards.

Proof (sketch). We first define the generic group model setting and all oracle interactions, including the oracles for encryption and token generation.

Generic group model. Let $\phi_{1}, \phi_{2}, \phi_{T}$ be distinct random injective mappings from the domain $\mathbb{Z}_{p}$ to $\{0,1\}^{\kappa}$, where $\kappa>3 \log p$. We write $\mathbb{G}_{1}$ for $\left\{\phi_{1}(x) \mid\right.$ $\left.x \in \mathbb{Z}_{p}\right\}, \mathbb{G}_{2}$ for $\left\{\phi_{2}(x) \mid x \in \mathbb{Z}_{p}\right\}$, and $\mathbb{G}_{T}$ for $\left\{\phi_{T}(x) \mid x \in \mathbb{Z}_{p}\right\}$. The adversary is given access to an oracle to compute the group actions on $\mathbb{G}_{1}, \mathbb{G}_{2}$, and $\mathbb{G}_{T}$. Additionally, it is given access to an oracle capable of computing a non-degenerate bilinear map $e: \mathbb{G}_{1} \times \mathbb{G}_{2} \rightarrow \mathbb{G}_{T}$. Lastly, we also define a random oracle to model the hash function $H:\{0,1\} \rightarrow \mathbb{G}_{1}$.

Instead of writing $\phi_{1}(x)$, we write $g_{1}^{x}$. Similarly, we write $g_{2}^{x}$ for $\phi_{2}(x)$ and $e\left(g_{1}, g_{2}\right)^{x}$ for $\phi_{T}(x)$.

Hash oracle $H$. The challenger keeps track of oracle queries it received before by maintaining a table. If it has not received an oracle query for the value id before, it chooses a random value $t_{\text {id }} \in \mathbb{Z}_{p}$ and stores this value in its table. It returns the value $g_{1}^{t_{\text {id }}}$ to the querier.

Game interactions. The adversary's first interaction with the challenger is to receive the group parameters and the secret keys of the corrupted clients.

Setup The challenger chooses $\alpha_{i}, \gamma_{i} \stackrel{R}{\leftarrow} \mathbb{Z}_{p}^{*}$ and $\beta_{i} \stackrel{R}{\leftarrow} \mathcal{K}$ for $1 \leq i \leq n$, just like in the actual scheme. It also defines the secret keys usk ${ }_{i}$ and master secret key msk according to the scheme.

Corruptions The adversary submits its choices for the corrupted clients $\bar{I}$ to the challenger. In the selective plaintext-privacy game, the adversary additionally submits its challenge inputs $\left(\mathrm{id}^{*}, \boldsymbol{x}_{0, I}^{*}, \boldsymbol{x}_{1, I}^{*}\right)$. The challenger gives the secret keys usk $_{\bar{I}}$ of the corrupted clients to the adversary. 
Queries The adversary interacts with the challenger by asking the challenger to encrypt a messages or to generate a token for some predicate. To be able to refer to a specific query later on in the proof, we label every query with a query number. Let $j$ represent this query number.

Encrypt The challenger answers valid Encrypt queries for a message $x_{i}^{(j)}$ for client $i$ and identifier id ${ }^{(j)}$ similar as in the scheme. It chooses $r_{i}^{(j)} \stackrel{R}{\leftarrow} \mathbb{Z}_{p}^{*}$ and returns the ciphertext $\mathrm{ct}_{i, \mathrm{id}}^{(j)}$,

$$
\left(g_{1}^{t_{\mathrm{id}}(j)}, g_{1}^{r_{i}^{(j)}}, g_{1}^{\alpha_{i} \pi\left(\beta_{i}, x_{i}^{(j)}\right) r_{i}^{(j)}} g_{1}^{t_{\mathrm{id}(j)} \gamma_{i}}\right)
$$

GenToken ${ }^{\star}$ Token queries for $\boldsymbol{y}^{(j)}$ are answered according to the scheme as well. The challenger chooses $u_{i}^{(j)} \stackrel{R}{\leftarrow} \mathbb{Z}_{p}^{*}$ for $i \in S_{\boldsymbol{y}^{(j)}}$ and returns the token $\mathrm{tk}_{\boldsymbol{y}}^{(j)}$,

$$
\left(\left\{g_{2}^{u_{i}^{(j)}}, g_{2}^{\alpha_{i} \pi\left(\beta_{i}, y_{i}^{(j)}\right) u_{i}^{(j)}} \mid i \in S_{\boldsymbol{y}}\right\}, \prod_{i \in S_{\boldsymbol{y}}} g_{2}^{u_{i}^{(j)} \gamma_{i}}\right)
$$

to the adversary.

Proof structure. We prove both selective plaintext privacy and restricted adaptive predicate privacy through a series of hybrid games.

For selective plaintext privacy the number of games depends on the number of differentiating components of the challenge inputs - hence the selective game type. Let $\bar{X}$ denote the set of indices where the components of $\boldsymbol{x}_{0}^{*}$ differ from $\boldsymbol{x}_{1}^{*}$, $\bar{X}=\left\{i \mid x_{0, i}^{*} \neq x_{1, i}^{*}\right\}$. Let game $k$ be identical to the original game, except that in the challenge phase now the first $k-1$ components of $\bar{X}$ in the returned challenge vector are chosen at random. Note that game $k=1$ is identical to the original game and that in game $k=|\bar{X}|$ not even an unbounded adversary is able to gain an advantage in winning the game.

For restricted adaptive predicate privacy, we assume w.l.o.g. that $\boldsymbol{y}_{0, I}^{*} \neq \boldsymbol{y}_{1, I}^{*}$, because if $\boldsymbol{y}_{0, I}^{*}=\boldsymbol{y}_{1, I}^{*}$, the adversary would not be able to gain an advantage in the game since this implies $\boldsymbol{y}_{0}^{*}=\boldsymbol{y}_{1}^{*}$. Note that this means that the result of Match ${ }^{\star}$ with any allowed ciphertext vector will be FALSE. We define game $k$ identical to the original game, except that in the challenge phase now the first $k-$ 1 components of the returned challenge vector are chosen at random. Note that game $k=1$ is identical to the original game and that in game $k=n$ not even an unbounded adversary is able to gain an advantage in winning the game.

For both the selective plaintext-privacy as well as the restricted adaptive predicate-privacy game, we show that an adversary has at most an advantage 
of $O\left(q^{2} / p\right)$ in distinguishing between game $k$ and game $k+1$. Furthermore, we use another hybrid game to change to a real-or-random based challenge instead of a left-or-right based challenge. It is not difficult to see that an adversary gaining an advantage $\epsilon$ in the left-or-right based game, gains an advantage of at least $\frac{\epsilon}{2}$ in the real-or-random based game.

Challenges. Since we changed the game to a real-or-random based game, the challenge phase changes slightly. The challenger now chooses a bit $b \stackrel{R}{\leftarrow}\{0,1\}$ that is used to determine whether to return the encryption of the submitted value or a random one. In case of the selective plaintext-privacy game, the adversary submits a vector $\boldsymbol{x}_{I}^{(c)}$ together with an identifier id ${ }^{(c)}$ to the challenger. In case of the restricted predicate-privacy game, the adversary submits a vector $\boldsymbol{y}^{(c)}$ to the challenger. The challenger chooses values $\nu_{i}, \nu_{i}^{\prime} \stackrel{R}{\leftarrow} \mathbb{Z}_{p}^{*}$ for $1 \leq i \leq n$. For a ciphertext challenge it returns the challenge

$$
\mathrm{ct}_{\mathrm{Ch}}=\left\{\left(g_{1}^{t_{\mathrm{id}(c)}}, g_{1}^{\nu_{i}}, \mathrm{ct}_{\mathrm{Ch}, i}^{\prime}\right) \mid i \in I\right\}
$$

where

$$
\mathrm{ct}_{\mathrm{Ch}, k}^{\prime}= \begin{cases}g_{1}^{\nu_{k} \alpha_{k} \pi\left(\beta_{k}, \nu_{k}^{\prime}\right)+t_{\mathrm{id}(c)} \gamma_{k}} & \text { if } b=0 \\ g_{1}^{\nu_{k} \alpha_{k} \pi\left(\beta_{k}, x_{k}^{(c)}\right)+t_{\mathrm{id}}(c)} \gamma_{k} & \text { if } b=1 .\end{cases}
$$

For a token challenge, it returns the challenge

$$
\mathrm{tk}_{\mathrm{Ch}}=\left(\left\{\left(g_{2}^{\nu_{i}}, \mathrm{tk}_{\mathrm{Ch}, i}^{\prime}\right) \mid i \in S_{\boldsymbol{y}}\right\}, \prod_{i \in S_{\boldsymbol{y}}} g_{2}^{\nu_{i} \gamma_{i}}\right),
$$

where, if $k \in S_{\boldsymbol{y}}$,

$$
\mathrm{tk}_{\mathrm{Ch}, k}^{\prime}= \begin{cases}g_{2}^{\nu_{k} \alpha_{k} \pi\left(\beta_{k}, \nu_{k}^{\prime}\right)} & \text { if } b=0 \\ g_{2}^{\nu_{k} \alpha_{k} \pi\left(\beta_{k}, y_{k}^{(c)}\right)} & \text { if } b=1\end{cases}
$$

Indistinguishability. We now show that an adversary has at most a negligible advantage of $O\left(q^{2} / p\right)$ in distinguishing between game $k$ and game $k+1$, i.e., it is unable to distinguish $g_{1}^{\nu_{k} \alpha_{k} \pi\left(\beta_{k}, x_{k}^{(c)}\right)+t_{\text {id }(c)} \gamma_{k}}$ from $g_{1}^{\nu_{k}^{\prime}}$ for ciphertext challenges and $g_{2}^{\nu_{k} \alpha_{k} \pi\left(\beta_{k}, y_{k}^{(c)}\right)}$ from $g_{2}^{\nu_{k}^{\prime}}$ for token challenges.

As is common in the generic bilinear group model [Sho97], we consider the challenger keeping record of all group elements the adversary has. It does so by keeping lists $P_{\mathbb{G}, l}$ of linear polynomials in $\mathbb{Z}_{p}$ for each of the groups $\mathbb{G}_{1}, \mathbb{G}_{2}$, 
and $\mathbb{G}_{T}$. These polynomials use indeterminates for $\gamma_{i}, \alpha_{i} \pi\left(\beta_{i}, c_{i}\right)$, the $t_{\mathrm{id}^{(j)}}$ 's, $\alpha_{i} \pi\left(\beta_{i}, x_{i}^{(j)}\right)$ 's, $\alpha_{i} \pi\left(\beta_{i}, y^{(j)}\right)$ 's, $r_{i}^{(j)}$ 's, and the $u_{i}^{(j)}$ 's.

To simplify our reasoning, we will only look at polynomials $P_{\mathbb{G}_{T}, l}$ in $\mathbb{G}_{T}$. This is justified as we can transform any polynomial in $\mathbb{G}_{1}$ or $\mathbb{G}_{2}$ to a polynomial $P_{\mathbb{G}_{T}, l}$ in $\mathbb{G}_{T}$ through an additional query to the pairing oracle.

We can now say that the adversary wins the game if for a random assignment to all the indeterminates, any $P_{\mathbb{G}_{T}, i} \neq P_{\mathbb{G}_{T}, j}$ evaluates to the same value. We will show that the adversary is not able to query for distinct polynomials $P_{\mathbb{G}_{T}, i}$, $P_{\mathbb{G}_{T}, j}$ such that, if the challenger plays the 'real' experiment and if the indeterminates get assigned with random values, they will evaluate to the same value, except for negligible probability. Then, by the Schwartz lemma [Sch80] and the extended result of Shoup [Sho97], we can bound this probability of $P_{\mathbb{G}_{T}, i} \neq P_{\mathbb{G}_{T}, j}$ evaluating to the same value by $O\left(q^{2} / p\right)$ if at most $q$ group elements are given to the adversary.

In the case of a ciphertext challenge, we first have to bring the challenge response, which is an element of $\mathbb{G}_{1}$, to the target group $\mathbb{G}_{T}$. Since the adversary only has (linear combinations of) the elements $g_{2}, g_{2}^{u_{i}^{(j)}}, g_{2}^{\alpha_{i} \pi\left(\beta_{i}, y_{i}^{(j)}\right) u_{i}^{(j)}}$, and $\prod_{i \in S_{y}} g_{2}^{u_{i}^{(j)} \gamma_{i}}$ in $\mathbb{G}_{2}$, it can only bring the challenge to $\mathbb{G}_{T}$ by pairing with one of these. Similarly, for token challenges, the adversary can only pair with the elements $g_{1}, g_{1}^{t_{\mathrm{id}}(j)}, g_{1}^{r_{i}^{(j)}}$, or $g_{1}^{\alpha_{i} \pi\left(\beta_{i}, x_{i}^{(j)}\right) r_{i}^{(j)}+t_{\mathrm{id}(j)} \gamma_{i}}$ in $\mathbb{G}_{1}$.

The resulting polynomials for these challenge responses are summarized in Table 1. Since the group elements are represented by uniformly independent values, the adversary can only distinguish between game $k$ and game $k+1$ with more than a negligible advantage if it can construct at least one of the polynomials in this table.

Linear combinations. We now argue that the adversary cannot construct any of these challenges by looking at the components it has. We summarize the polynomials the adversary has access to, again by only looking at the elements in the target group $\mathbb{G}_{T}$, in Table 2 . We have to show that no linear combination of the polynomials in Table 2 equals any of the polynomials in Table 1 .

First, we look at the target queries for a ciphertext challenge to prove selective plaintext privacy and restricted adaptive predicate privacy without static corruptions. Later, we will explain that the construction is indistinguishable if we allow for static corruptions as well. 
Table 1: Target polynomials in both indistinguishability games.

Ciphertext challenge

\begin{tabular}{l|l}
\hline$\nu_{k} \alpha_{k} \pi\left(\beta_{k}, x_{k}^{(c)}\right)+t_{\text {id }(c)} \gamma_{k}$ & $u_{i}^{(j)}\left(\nu_{k} \alpha_{k} \pi\left(\beta_{k}, x_{k}^{(c)}\right)+t_{\text {id }(c)} \gamma_{k}\right)$ \\
\hline$u_{i}^{(j)} \alpha_{i} \pi\left(\beta_{i}, y_{i}^{(j)}\right)\left(\nu_{k} \alpha_{k} \pi\left(\beta_{k}, x_{k}^{(c)}\right)+t_{\text {id }(c)} \gamma_{k}\right)$ & $\left(\nu_{k} \alpha_{k} \pi\left(\beta_{k}, x_{k}^{(c)}\right)+t_{\mathrm{id}^{(c)}} \gamma_{k}\right) \sum_{i \in S_{y^{(j)}}} u_{i}^{(j)} \gamma_{i}$
\end{tabular}

Token challenge

\begin{tabular}{l|l}
\hline$\nu_{k} \alpha_{k} \pi\left(\beta_{k}, y_{k}^{(c)}\right)$ & $t_{\mathrm{id}} \nu_{k} \alpha_{k} \pi\left(\beta_{k}, y_{k}^{(c)}\right)$ \\
\hline$r_{i}^{(j)} \nu_{k} \alpha_{k} \pi\left(\beta_{k}, y_{k}^{(c)}\right)$ & $\left(r_{i}^{(j)} \alpha_{i} \pi\left(\beta_{i}, x_{i}^{(j)}\right)+t_{\text {id }} \gamma_{i}\right) \nu_{k} \alpha_{k} \pi\left(\beta_{k}, y_{k}^{(c)}\right)$
\end{tabular}

Table 2: Elements the adversary can query for in an indistinguishability game (up to linear combinations).

\begin{tabular}{|c|c|c|c|}
\hline 1 & $t_{\mathrm{id}^{(j)}}$ & $r_{i}^{(j)}$ & $r_{i}^{(j)} \alpha_{i} \pi\left(\beta_{i}, x_{i}^{(j)}\right)+t_{\mathrm{id}(j)} \gamma_{i}$ \\
\hline$\overline{u_{i^{\prime}}^{\left(j^{\prime}\right)}}$ & $u_{i^{\prime}}^{\left(j^{\prime}\right)} t_{\mathrm{id}(j)}$ & $u_{i^{\prime}}^{\left(j^{\prime}\right)} r_{i}^{(j)}$ & $u_{i^{\prime}}^{\left(j^{\prime}\right)}\left(r_{i}^{(j)} \alpha_{i} \pi\left(\beta_{i}, x_{i}^{(j)}\right)+t_{\mathrm{id}(j)} \gamma_{i}\right)$ \\
\hline$\overline{u_{i^{\prime}}^{\left(j^{\prime}\right)} \alpha_{i^{\prime}} \pi\left(\beta_{i^{\prime}}, y_{i^{\prime}}^{\left(j^{\prime}\right)}\right)}$ & $u_{i^{\prime}}^{\left(j^{\prime}\right)} \alpha_{i^{\prime}} \pi\left(\beta_{i^{\prime}}, y_{i^{\prime}}^{\left(j^{\prime}\right)}\right) t_{\mathrm{id}^{(j)}}$ & $u_{i^{\prime}}^{\left(j^{\prime}\right)} \alpha_{i^{\prime}} \pi\left(\beta_{i^{\prime}}, y_{i^{\prime}}^{\left(j^{\prime}\right)}\right) r_{i}^{(j)}$ & $u_{i^{\prime}}^{\left(j^{\prime}\right)} \alpha_{i^{\prime}} \pi\left(\beta_{i^{\prime}}, y_{i^{\prime}}^{\left(j^{\prime}\right)}\right)\left(r_{i}^{(j)} \alpha_{i} \pi\left(\beta_{i}, x_{i}^{(j)}\right)+t_{\mathrm{id}(j)} \gamma_{i}\right)$ \\
\hline$\sum_{i^{\prime} \in S_{\boldsymbol{y}\left(j^{\prime}\right)}} u_{i^{\prime}}^{\left(j^{\prime}\right)} \gamma_{i^{\prime}}$ & $t_{\mathrm{id}^{(j)}} \sum_{i^{\prime} \in S_{\boldsymbol{y}^{\left(j^{\prime}\right)}}} u_{i^{\prime}}^{\left(j^{\prime}\right)} \gamma_{i^{\prime}}$ & $r_{i}^{(j)} \sum_{i^{\prime} \in S_{y}\left(j^{\prime}\right)} u_{i^{\prime}}^{\left(j^{\prime}\right)} \gamma_{i^{\prime}}$ & $\left(r_{i}^{(j)} \alpha_{i} \pi\left(\beta_{i}, x_{i}^{(j)}\right)+t_{\mathrm{id}^{(j)}} \gamma_{i}\right) \sum_{i^{\prime} \in S_{\boldsymbol{y}^{\left(j^{\prime}\right)}}} u_{i^{\prime}}^{\left(j^{\prime}\right)} \gamma_{i^{\prime}}$ \\
\hline
\end{tabular}


Plaintext privacy. Observe that all polynomials for the ciphertext challenges contain $t_{\mathrm{id}(c)} \gamma_{k}$. This means that we only have to consider the forth column of Table 2 and the polynomial $t_{\text {id }} \sum_{i^{\prime} \in S_{y^{\left(j^{\prime}\right)}}} u_{i^{\prime}}^{\left(j^{\prime}\right)} \gamma_{i^{\prime}}$. However, we require $t_{\mathrm{id}(j)}=t_{\mathrm{id}(c)}$, but we do not have any of the elements in the fourth column like this: If $t_{\mathrm{id}^{(j)}}=t_{\mathrm{id}(c)}$, the adversary has requested an illegal query by using the challenge identifier id ${ }^{(c)}$ in a query phase. Therefore, only the polynomial $t_{\text {id }(c)} \sum_{i^{\prime} \in S_{y^{\left(j^{\prime}\right)}}} u_{i^{\prime}}^{\left(j^{\prime}\right)} \gamma_{i^{\prime}}$ can be used, where the token query for $\boldsymbol{y}^{\left(j^{\prime}\right)}$ does not contain a wildcard in position $k$. This, in its turn, implies that the target ciphertext challenge has to contain exactly $t_{\mathrm{id}(c)} u_{k}^{\left(j^{\prime}\right)} \gamma_{k}$. Let $\boldsymbol{y}^{(\lambda)}$ be an arbitrary queried vector such that $y_{k}^{(\lambda)} \neq \star$. Now, by looking at Table 1 , we conclude that we are left proving that no linear combination of the polynomials in Table 2 can form either $u_{k}^{(\lambda)}\left(\nu_{k} \alpha_{k} \pi\left(\beta_{k}, x_{k}^{(c)}\right)+t_{\text {id }^{(c)}} \gamma_{k}\right)$ or $u_{k}^{(\lambda)} \alpha_{k} \pi\left(\beta_{k}, y_{k}^{(\lambda)}\right)\left(\nu_{k} \alpha_{k} \pi\left(\beta_{k}, x_{k}^{(c)}\right)+t_{\text {id }}(c) \gamma_{k}\right)$.

The polynomial $t_{\text {id } d^{(c)}} u_{k}^{(\lambda)} \gamma_{k}$ can be constructed using $t_{\text {id }(c)} \sum_{i \in S_{y^{(\lambda)}} \backslash\{k\}} u_{i}^{(\lambda)} \gamma_{i}$. Now, to cancel this summed term, we look at Table 2 and see that summing $u_{i^{\prime}}^{\left(j^{\prime}\right)}\left(r_{i}^{(j)} \alpha_{i} \pi\left(\beta_{i}, x_{i}^{(j)}\right)+t_{\mathrm{id}(j)} \gamma_{i}\right)$ for $j=c, j^{\prime}=\lambda$, and $i=i^{\prime}$ is the only suitable polynomial. However, this introduces a new term $\sum_{i \in S_{\boldsymbol{y}}(\lambda) \backslash\{k\}} u_{i}^{(\lambda)} \nu_{i} \alpha_{i} \pi\left(\beta_{i}, x_{i}^{(c)}\right)$. In Table 2 we only find $u_{i^{\prime}}^{\left(j^{\prime}\right)} \alpha_{i^{\prime}} \pi\left(\beta_{i^{\prime}}, y_{i^{\prime}}^{\left(j^{\prime}\right)}\right) r_{i}^{(j)}$ that can be used to cancel this new term. Setting $j=c, j^{\prime}=\lambda$, and $i=i^{\prime}$, and summing results in

$$
\sum_{i \in S_{\boldsymbol{y}^{(\lambda)} \backslash\{k\}}} u_{i}^{(\lambda)} \alpha_{i} \pi\left(\beta_{i}, y_{i}^{(\lambda)}\right) \nu_{i} .
$$

Therefore, if the adversary queried for a token $\boldsymbol{y}^{(\lambda)}$ where index $k$ is not a wildcard and where

$$
y_{i}^{(\lambda)}=x_{i}^{(c)} \text { for all } i \in S_{\boldsymbol{y}^{(\lambda)}} \backslash\{k\},
$$

it can cancel this most recent introduced term as well. Now, the adversary has constructed the polynomial $u_{k}^{(\lambda)} t_{\text {id }(c)} \gamma_{k}$ and so it is left to construct either $u_{k}^{(\lambda)} \nu_{k} \alpha_{k} \pi\left(\beta_{k}, x_{k}^{(c)}\right)$ or $u_{k}^{(\lambda)} \alpha_{k} \pi\left(\beta_{k}, y_{k}^{(\lambda)}\right) \nu_{k} \alpha_{k} \pi\left(\beta_{k}, x_{k}^{(c)}\right)$. We claim that neither is possible.

Looking at Table 2 again, we see that only polynomial $u_{i^{\prime}}^{\left(j^{\prime}\right)} \alpha_{i^{\prime}} \pi\left(\beta_{i^{\prime}}, y_{i^{\prime}}^{\left(j^{\prime}\right)}\right) r_{i}^{(j)}$, for $j=c, j^{\prime}=\lambda, i=i^{\prime}=k$, and $y_{k}^{(\lambda)}=x_{k}^{(c)}$, is suitable to construct the polynomial $u_{k}^{(\lambda)} \nu_{k} \alpha_{i} \pi\left(\beta_{i}, x_{k}^{(c)}\right)$. This means that we require both Equation (1) and $y_{k}^{(\lambda)}=x_{k}^{(c)}$ to hold. However, this implies that Match ${ }^{\star}\left(\boldsymbol{x}^{(c)}, \boldsymbol{y}^{(\lambda)}\right)=$ TRUE and such a query for vector $\boldsymbol{y}^{(\lambda)}$ is not allowed in the real-or-random game.

We try to construct the polynomial $u_{k}^{(\lambda)} \alpha_{k} \pi\left(\beta_{k}, y_{k}^{(\lambda)}\right) \nu_{k} \alpha_{k} \pi\left(\beta_{k}, x_{k}^{(c)}\right)$ by looking at Table 22 We find the polynomial $u_{i^{\prime}}^{\left(j^{\prime}\right)} \alpha_{i^{\prime}} \pi\left(\beta_{i^{\prime}}, y_{i^{\prime}}^{\left(j^{\prime}\right)}\right)\left(r_{i}^{(j)} \alpha_{i} \pi\left(\beta_{i}, x_{i}^{(j)}\right)+\right.$ 
$\left.t_{\mathrm{id}^{(j)}} \gamma_{i}\right)$ for $j=c, j^{\prime}=\lambda, i=i^{\prime}=k$ as the only suitable candidate to cancel the term. This, however, introduces the new term $u_{k}^{(\lambda)} \alpha_{k} \pi\left(\beta_{k}, y_{k}^{(\lambda)}\right) t_{\mathrm{id}(c)} \gamma_{k}$, which can only be canceled by $u_{k}^{(\lambda)} \alpha_{k} \pi\left(\beta_{k}, y_{k}^{(\lambda)}\right)\left(\nu_{k} \alpha_{k} \pi\left(\beta_{k}, x_{k}^{(c)}\right)+t_{\text {id }_{(c)}} \gamma_{k}\right)$ again. This reintroduces the original term that we wanted to construct. This means that we always have a term that we cannot cancel out, therefore we conclude that the adversary cannot construct this polynomial either.

Combining all results, we conclude that there is no linear combination and thus that the construction is selective plaintext private without static corruptions.

Restricted adaptive predicate privacy. We now look at the target queries for a token challenge. We can only construct $\nu_{k} \alpha_{k} \pi\left(\beta_{k}, y_{k}^{(c)}\right)$ using $u_{k}^{\left(j^{\prime}\right)} \alpha_{k} \pi\left(\beta_{k}, x_{k}^{(j)}\right)$, where we require $j^{\prime}=c$ and $x_{k}^{(j)}=y_{k}^{(c)}$. These polynomials only occur in the last column of Table 2 However, the first cell in the column cannot be used as it does not contain indeterminates of the type $u$. The third and last cell, on the other hand, contain indeterminates that the adversary does not have access to, $\alpha_{k} \pi\left(\beta_{k}, y_{k}^{(c)}\right)$, or can never cancel, $\gamma_{i} \gamma_{i^{\prime}}$. Therefore, only the second cell for $i=i^{\prime}=k$ and $j^{\prime}=c, \nu_{k}\left(r_{k}^{(j)} \alpha_{k} \pi\left(\beta_{k}, x_{k}^{(j)}\right)+t_{\mathrm{id}^{(j)}} \gamma_{k}\right)$, seems to be a suitable candidate.

Let the $\lambda$ th query be an arbitrary ciphertext query where $x_{k}^{(\lambda)}=y_{k}^{(c)}$, now if we use this polynomial, we introduce the new term $\nu_{k} t_{\mathrm{id}(\lambda)} \gamma_{k}$. Looking at Table 2 we see that the only way to cancel this term is to use $t_{\mathrm{id}(\lambda)} \sum_{i \in S_{y^{(c)}}} u_{i}^{(c)} \gamma_{i}$. The additionally introduced terms can be canceled with $u_{i^{\prime}}^{\left(j^{\prime}\right)}\left(r_{i}^{(j)} \alpha_{i} \pi\left(\beta_{i}, x_{i}^{(j)}\right)+\right.$ $\left.t_{\mathrm{id}^{(j)}} \gamma_{i}\right)$ for $i=i^{\prime}, j=\lambda$, and $j^{\prime}=c$. However, this again introduces the polynomial $\sum_{i \in S_{y^{(c)}} \backslash\{k\}} u_{i}^{(c)} r_{i}^{(\lambda)} \alpha_{i} \pi\left(\beta_{i}, x_{i}^{(\lambda)}\right)$, which can only be canceled by $u_{i^{\prime}}^{\left(j^{\prime}\right)} \alpha_{i^{\prime}} \pi\left(\beta_{i^{\prime}}, y_{i^{\prime}}^{\left(j^{\prime}\right)}\right) r_{i}^{(j)}$ for $i=i^{\prime}$ and $j^{\prime}=\lambda$, where we require $x_{i}^{(\lambda)}=y_{i}^{(c)}$ for all $i \in S_{\boldsymbol{y}^{(c)} \backslash\{k\}}$. Combining this requirement with the requirement we made earlier, that $x_{k}^{(\lambda)}=y_{k}^{(c)}$, means that the adversary has to query for a ciphertext $\boldsymbol{x}^{(\lambda)}$ such that Match ${ }^{\star}\left(\boldsymbol{x}^{(\lambda)}, \boldsymbol{y}^{(c)}\right)=$ TRUE. Since this is not allowed in the game, we conclude that no token challenge can be made from a linear combination of the elements the adversary can query for.

Corruptions. We claim that, since we have proven the construction secure without corruptions and since the construction uses distinct, uncorrelated, encryptions keys usk ${ }_{i}$ for every client $i$, the construction is also secure under static corruptions. To see this, observe that the indistinguishability notion under static corruptions only guarantees indistinguishability of the uncorrupted vector components. This means that we can ignore the corrupted vector components and only require the adversary cannot learn the encryption key of client $j$ if it learned 
the encryption key of client $i$.

\section{A.2 Chosen-Plaintext Security}

The proposed construction is also chosen-plaintext secure as stated in Theorem 2 . We remark that the proof does not rely on the use of random oracles.

Proof. We construct a challenger $\mathcal{B}$ capable of breaking the DDH assumption in $\mathbb{G}_{1}$ by using an adversary $\mathcal{A}$ that is able to win the chosen-plaintext with corruptions game with more than a negligible advantage.

We proof this though a series of hybrid games. Let game $j$ be the game as defined in Definition 6, but where the first $j-1$ components of the challenge query are replaced by random elements. Note that game 1 is identical to the original game and that it is not possible for any adversary to gain an advantage in game $n+1$. We are left to show that an adversary has at most a negligible advantage in distinguishing game $j$ from game $j+1$.

Setup The challenger $\mathcal{B}$ receives the bilinear group parameters and the DDH instance $\left(A=g_{1}^{a}, B=g_{1}^{b}, Z\right) \in\left(\mathbb{G}_{1}\right)^{3}$. It chooses the hash function $H$ and the encryption keys usk ${ }_{i}$. It sets encryption key usk ${ }_{j}=\left(A, \beta_{j} \stackrel{R}{\leftarrow} \mathcal{K}, \gamma_{j} \stackrel{R}{\leftarrow} \mathbb{Z}_{p}^{*}\right)$ and chooses the rest of the encryption keys according to the scheme. The public parameters and the encryption keys usk ${ }_{i}$ are given to the adversary.

Challenge The adversary $\mathcal{A}$ submits an identifier id* and two vectors $\boldsymbol{x}_{0}^{*}$, $\boldsymbol{x}_{1}^{*}$ to the challenger. The challenger chooses $b \stackrel{R}{\leftarrow}\{0,1\}$ and sets $g_{1}^{r_{j}}=B$. Additionally, it picks values $r_{i} \stackrel{R}{\leftarrow} \mathbb{Z}_{p}^{*}$ for $1 \leq i \neq j \leq n$. It gives the challenge

$$
\mathrm{ct}_{i}= \begin{cases}\left(H\left(\mathrm{id}^{*}\right), g_{1}^{r_{i}}, R \stackrel{R}{\leftarrow} \mathbb{G}_{1}\right) & \text { if } i<j \\ \left(H\left(\mathrm{id}^{*}\right), B, Z^{\pi\left(\beta_{i}, x_{b, i}^{*}\right)} H\left(\mathrm{id}^{*}\right)^{\gamma_{j}}\right) & \text { if } i=j \\ \left(H\left(\mathrm{id}^{*}\right), g_{1}^{r_{i}}, g_{1}^{\alpha_{i} r_{i} \pi\left(\beta_{i}, x_{b, i}^{*}\right)} H\left(\mathrm{id}^{*}\right)^{\gamma_{i}}\right) & \text { if } i>j\end{cases}
$$

for $1 \leq i \leq n$ to the adversary.

If the challenger is given $Z=g_{1}^{a b}$, then challenge ciphertext is identically distributed as the challenge ciphertext in game $j$ and component $j$ is a real encryption. If the challenger is given $Z \stackrel{R}{\leftarrow} \mathbb{G}_{1}$, then challenge ciphertext is identically distributed as the challenge ciphertext in game $j+1$ and component $j$ is a random encryption.

Guess The challenger outputs its guess that $Z=g_{1}^{a b}$ if the adversary guesses that it is playing game $j$, and outputs its guess that $Z \stackrel{R}{\leftarrow} \mathbb{G}_{1}$ if the adversary guesses that it is playing game $j+1$.

If the adversary has a non-negligible advantage in distinguishing between game $j$ and game $j+1$, the challenger obtains a non-negligible advantage in solving the DDH problem in group $\mathbb{G}_{1}$. 